\title{
Exposure to zinc deficiency in fetal and postnatal life determines nitric oxide system activity and arterial blood pressure levels in adult rats
}

\author{
Analía Tomat ${ }^{1}$, Rosana Elesgaray ${ }^{1}$, Valeria $\mathrm{Zago}^{2}$, Héctor Fasoli ${ }^{3}$, Andrea Fellet $^{1}$, Ana María Balaszczuk ${ }^{1}$, \\ Laura Schreier ${ }^{2}$, María Ángeles Costa ${ }^{1}$ and Cristina Arranz ${ }^{1} *$ \\ ${ }^{1}$ Cátedra de Fisiología, Facultad de Farmacia y Bioquímica, Universidad de Buenos Aires, IQUIMEFA-CONICET, Junín 956, \\ piso 7, Ciudad Autónoma de Buenos Aires, Argentina \\ ${ }^{2}$ Laboratorio de Lípidos y Lipoproteínas, Dto. Bioquímica Clínica, Facultad de Farmacia y Bioquímica, Universidad de Buenos \\ Aires, Junín 956, Ciudad Autónoma de Buenos Aires, Argentina \\ ${ }^{3}$ Laboratorio de Química y Ciencia Ambiental, Facultad de Ciencias Fisicomatemáticas e Ingeniería, Universidad Católica \\ Argentina, Alicia M. de Justo 1500, Buenos Aires, Argentina
}

(Received 4 September 2009 - Revised 9 February 2010 - Accepted 10 February 2010 - First published online 26 March 2010)

\begin{abstract}
We had previously shown that prenatal exposure to $\mathrm{Zn}$-deficient diets induces an increase in blood pressure and impairs renal function in adult rats. The aim of the present study was to investigate if moderate $\mathrm{Zn}$ restriction during early growth periods, fetal life and lactation would induce impairment in the vascular and renal NO system and alterations in plasma lipid profile. We also investigated if these effects persisted into adult life, even when a $\mathrm{Zn}$-replete diet was provided after weaning. Pregnant rats were fed control (30 parts per million (ppm)) or low ( $8 \mathrm{ppm}) \mathrm{Zn}$ diets throughout gestation up to weaning. Afterwards, male offspring from low-Zn mothers were assigned to low- or control-Zn diets during $60 \mathrm{~d}$. Male offspring from control mothers were fed a control diet. Animals exposed to Zn restriction showed low birth weight, increased systolic blood pressure and serum TAG levels, and decreased glomerular filtration rate in adulthood. Zn restriction induced a decrease in vascular and renal NO synthase activity and a reduced expression of the endothelial NO synthase isoform in aorta. A control-Zn diet during post-weaning growth returned TAG levels to normal but was unsuccessful in normalising systolic blood pressure, glomerular filtration rate or NO system activity in Zn-deficient offspring. Zn restriction during fetal life, lactation and/or post-weaning growth induced alterations in the vascular and renal NO system and in lipid metabolism that could contribute to the programming of hypertension and renal dysfunction in adulthood.
\end{abstract}

Arterial blood pressure: Nitric oxide system: Moderate zinc restriction: Fetal life

Human epidemiological and experimental studies have provided considerable evidence to suggest that nutritional imbalance and metabolic disturbances during critical developmental time windows have persistent effects on the health of the offspring and may be responsible for in utero programming of common disorders such as obesity, diabetes and hypertension in adult life ${ }^{(1-3)}$. Moderate and marginal $\mathrm{Zn}$ deficiency observed in pregnant women could be a nutritional insult to fetal and postnatal development ${ }^{(4,5)}$. Moreover, this micronutrient could program adult pathologies by an epigenetic mechanism since it controls methylation reactions and epigenetic modifications of DNA and histones ${ }^{(6,7)}$.

$\mathrm{Zn}$ is found in a wide variety of foods such as whole-grain cereals, legumes, meat, chicken and fish. However, moderate $\mathrm{Zn}$ deficiency is mostly due to nutritional imbalances in those stages of life when $\mathrm{Zn}$ requirements are increased, such as postnatal growth and pregnancy ${ }^{(5,8)}$.
We had previously reported that moderate $\mathrm{Zn}$ restriction during fetal life, lactation and/or post-weaning growth of rats induces an increase in arterial blood pressure (BP) and impairs renal function in adult life. These alterations were associated with an increase in renal oxidative stress, activation of renal apoptosis and fibrosis, and a reduction in the renal filtration surface area ${ }^{(9)}$. Moreover, we also reported that animals exposed to low $\mathrm{Zn}$ intake only since weaning and up to adult life showed an impairment in the vascular and renal NO system ${ }^{(10,11)}$. Up to now there has been no evidence about the influence of $\mathrm{Zn}$ deficiency during fetal life and lactation on this system.

It has been demonstrated that NO is an important factor in the regulation of blood flow and $\mathrm{BP}$ in mammals since it exerts a basal tonic relaxing action on systemic vasculature ${ }^{(12,13)}$. Moreover, NO plays a prominent role in the homeostatic regulation of glomerular, vascular and tubular functions

\footnotetext{
Abbreviations: BP, blood pressure; C, control Zn during pregnancy and lactation; Cc, control Zn during pregnancy, lactation and post-weaning; eNOS, endothelial NO synthase; GFR, glomerular filtration rate; iNOS, inducible NO synthase; L, low Zn during pregnancy and lactation; Lc, low Zn during pregnancy and lactation, control Zn post-weaning; Ll, low Zn during pregnancy, lactation and post-weaning; NADPH-d, NADPH diaphorase; nNOS, neuronal NO synthase; NOS, NO synthase; NOx, nitrites and nitrates; SBP, systolic BP.

* Corresponding author: Dr Cristina Teresa Arranz, fax +54 11 45083645, email carranz@ffyb.uba.ar
} 
in the kidney ${ }^{(14,15)}$. Therefore, it is likely that NO system impairment could be involved in the increase in BP levels and in the reduction in glomerular filtration rate (GFR) previously observed in rats exposed to $\mathrm{Zn}$ restriction during fetal and postnatal development ${ }^{(9)}$.

It is known that NO is a small gaseous molecule produced in vivo by NO synthase (NOS). The NOS family consists of three isoforms: neuronal (nNOS), endothelial (eNOS) and inducible (iNOS), which are expressed in many tissues, including endothelium and vascular smooth muscle and kidney. All NOS isoforms contain a zinc thiolate ( $\mathrm{ZnS} 4)$ cluster that plays an essential role in the catalytic activity of this enzyme by maintaining stability of the dimer interface and integrity of the tetrahydrobiopterin binding site ${ }^{(14,16)}$.

Moreover, $\mathrm{Zn}$ deficiency could be a cardiovascular risk factor associated with alterations in lipoprotein, cholesterol and TAG metabolism. It has been reported that severe $\mathrm{Zn}$ deficiency in adult rats induces increased fatty acid de novo synthesis, decreased fatty acid oxidation and reduced activity of lipoprotein lipase, which may contribute to an increase in TAG concentration ${ }^{(17,18)}$. Other authors have shown that severe $\mathrm{Zn}$ deficiency leads to a decrease in total and HDL-cholesterol, an increase in VLDL- and intermediate-density lipoprotein-cholesterol, or no changes in cholesterol lipoproteins ${ }^{(19-22)}$.

Therefore, the aim of the present study was to investigate whether moderate $\mathrm{Zn}$ restriction during early growth periods, fetal life and lactation would induce impairment in the vascular and renal total NOS activity and on the expression of the different NOS isoforms associated with high arterial BP levels and functional renal alterations in adult life. Moreover, we evaluated whether $\mathrm{Zn}$ deficiency induces alterations in plasma lipid profile, which is considered a cardiovascular risk factor. We also investigated if the effects of $\mathrm{Zn}$ restriction in utero and during lactation persisted into adult life, even when a Zn-replete diet was provided after weaning.

The present study used the same design as our previous study, but it was an independent experiment. Offspring, derived from a second set of dams, were fed similar diets and followed an identical experimental protocol as those used previously ${ }^{(9)}$.

\section{Materials and methods}

\section{Animals and study design}

Female Wistar rats from the breeding laboratories of the Facultad de Farmacia y Bioquímica (Universidad de Buenos Aires, Argentina) were mated by exposure to Wistar males during 1 week. Immediately afterwards, female rats were randomly fed either a moderately $\mathrm{Zn}$-deficient diet (L; 8 parts per million (ppm)) or a control-Zn diet $(\mathrm{C} ; 30 \mathrm{ppm})$ during the pregnancy and lactation periods. After birth, offspring were weighed and no more than nine rat pups remained with each mother. After weaning, male offspring from each $\mathrm{L}$ mother were randomly assigned to low- (8 ppm; Ll; $n$ 10) or control- (30 ppm; Lc; $n$ 10) $\mathrm{Zn}$ diets during $60 \mathrm{~d}$. Meanwhile, male offspring from $\mathrm{C}$ mothers were fed a control diet $(\mathrm{Cc})$.

Diet composition is outlined in Table 1. Both diets had the necessary nutrients, except $\mathrm{Zn}$ content, to meet rat
Table 1. Composition of the experimental diets ( $\mathrm{g} / \mathrm{kg}$ diet)

\begin{tabular}{lcc}
\hline Diet & Control diet & Low-Zn diet \\
\hline Potassium caseinate* & 200 & 200 \\
Soyabean oil & 70 & 70 \\
Mineral mix† & 35 & - \\
Zn-free salt mixture $\ddagger$ & - & 35 \\
Vitamin mix§ & 10 & 10 \\
Choline chloride & 1 & 1 \\
Maltodextrin & 684 & 684 \\
Zn & 0.03 & 0.008 \\
\hline
\end{tabular}

* Nestlé Argentina S.A., contaning $85.1 \mathrm{~g}$ protein/100 g.

† Mineral mix composition according to American Institute of Nutrition (AIN)-93 $\operatorname{diet}^{(23)}$.

$\ddagger$ Composition identical to mineral mix but without zinc chloride.

$\S$ Vitamin mix composition according to AIN-93 $\operatorname{diet}^{(23)}$.

requirements for the periods of pregnancy, lactation and growth according to American Institute of Nutrition (AIN)93 recommendations ${ }^{(23)}$.

Animals were allowed food and deionised water ad libitum. All laboratory material was previously washed with nitric acid (20\%) and deionised water. Male offspring were housed separately in plastic cages in a humidity- and temperaturecontrolled environment, with a $12 \mathrm{~h}$ light-dark cycle.

At day 56 after weaning, rats were fasted overnight, tail blood was collected and centrifuged and serum samples were stored at $-20^{\circ} \mathrm{C}$ until the moment of lipid analysis. TAG, total cholesterol and HDL-cholesterol were measured by standardised enzymic methods, under good quality-control conditions (Selectra 2 Vitalab analyser, multiple calibrator CEFAS; Roche Diagnostics GmbH, Mannheim, Germany). VLDL was determined in the supernatant fraction after LDL selective precipitation $^{(24)}$. Non-HDL-cholesterol, as an indicator of apoB-containing lipoproteins, was calculated as the difference between total cholesterol and HDL-cholesterol. Values for plasma lipids are fasting measurements in order to standardise results, given the great variability in these parameters.

Systolic BP (SBP) was measured indirectly in awake animals by the tail-cuff method using a Grass polygraph (model 79H; Grass Instrument Co., Quincy, MA, USA) at $60 \mathrm{~d}$ after weaning, as described previously ${ }^{(10)}$.

At day 60 after weaning, blood samples were collected from rats' tails and animals were placed in plastic metabolism cages in order to collect $24 \mathrm{~h}$ urine and faeces samples. Urine volume was determined gravimetrically. Plasma and urinary creatinine levels were measured by a colorimetric method (Wiener Laboratory, Rosario, Argentina). Creatinine clearance was calculated to estimate GFR. Nitrites and nitrates (NOx) were measured in urine samples by a colorimetric method, according to the procedure described by Verdon et al. ${ }^{(25)}$. $\mathrm{Zn}$ concentration in plasma, faeces, kidneys and diet was determined using an atomic absorption spectrophotometer (Varian Spectrophotometer Spectr AA-20, air acetylene flame, $0.5 \mathrm{~nm}$ slit, wavelength of $213.9 \mathrm{~nm}$; Perkin Elmer Corp., Norwalk, CT, USA), as described previously ${ }^{(10,11)}$.

At the end of the dietary treatment, rats were killed by cervical decapitation and the kidneys, the thoracic aorta artery and a segment of small intestine were immediately removed. 


\section{Nitric oxide synthase activity}

NOS activity was measured in the thoracic aorta artery and in the renal cortex of $\mathrm{Cc}$, $\mathrm{Lc}$ and $\mathrm{Ll}$ animals with $\mathrm{L}-\left[\mathrm{U}-{ }^{14} \mathrm{C}\right]$ arginine as the substrate (specific activity: $13319 \mathrm{MBq}$ (360 mCi)/mmol; Perkin Elmer Life and Analytical Sciences, Boston, MA, USA) using a liquid scintillation counter (Wallac 1414 WinSpectral; EG\&G Company, Turku, Finland), as described previously ${ }^{(26)}$.

\section{NADPH diaphorase activity}

The thoracic aorta artery, the kidney and a segment of the small intestine from $\mathrm{Cc}, \mathrm{Lc}$ and $\mathrm{Ll}$ rats were processed by the NADPH diaphorase (NADPH-d) histochemical method, as described previously ${ }^{(10,11,27)}$. This technique is used as a marker of isozyme-independent NOS.

The NADPH-d-stained cells from the different groups were measured using a Nikon E400 light microscope (Nikon Instrument Group, Melville, NY, USA) equipped with a digital camera connected to Image-Pro Plus 4.5.1.29 software (Media Cybernetics, LP, Silver Spring, MD, USA) and computerised acquisition and analysis software (Scion Image Beta 4.02; Scion Corporation, Walkersville, MD, USA). The mean of each optical density (OD) value was calculated by the measurement of OD in different tissue areas of the same section and in different sections of the same organ. Each set of OD measurements (control and experimental groups) was performed blindly and under similar light, gain, offset and magnification conditions.

\section{Western blot}

Samples of renal cortex and thoracic aorta containing equal amounts of protein $(10 \mu \mathrm{g}$ protein/lane) were separated by electrophoresis in $7.5 \%$ SDS-polyacrylamide gels (Bio-Rad, Munich, Germany), transferred to a nitrocellulose membrane (Bio-Rad), and then incubated with rabbit polyclonal antiNOS antibodies (Santa Cruz Biotechnology, Santa Cruz, CA, USA, dilution 1:500, anti-iNOS: epitope at the carboxy terminus, anti-eNOS: epitope at the amino terminus, and anti-nNOS: epitope at the amino terminus) and a secondary immunoreaction with a goat anti-rabbit antibody conjugated with horseradish peroxidase was performed (dilution 1:5000; Amersham Pharmacia Biotech, Uppsala, Sweden). Samples were revealed by chemiluminescence using ECL reagent for 2-4 min (Amersham Pharmacia Biotech). Quantification of the bands was performed by digital image analysis using a Hewlett-Packard scanner and TotalLab analyser software (Nonlinear Dynamics Ltd, Newcastle upon Tyne, UK). All experiments were performed in triplicate.

\section{Statistical analysis}

Values are means with their standard errors. Prism Graph Pad Software (San Diego, CA, USA) was used for statistical analysis. Data were analysed using one-way ANOVA followed by a Bonferroni multiple-comparison post hoc test. TAG were analysed by the non-parametric Dunn test for multiple comparisons. Linear regression analysis was used to determine the relationship between birth weight and SBP and NOS activity in the renal cortex and GFR at $60 \mathrm{~d} . P<0.05$ was considered a significant difference.

\section{Ethical approval for animal experimentation}

Animals were cared for according to regulation 6344/96 of Argentina's National Drug, Food and Medical Technology Administration and the Guide for the Care and Use of Laboratory Animals published by the US National Institutes of Health (NIH publication no. 85-23, revised 1996). Experimental procedures were approved by the ethics committee of the School of Biochemistry and Pharmacy (CEFFB), Buenos Aires University, Argentina.

\section{Results}

The mothers fed either a moderately Zn-deficient diet or a control diet exhibited similar daily food intake during the experimental period (L, 30.5 (SEM 3.7); C, 29.2 (SEM 2.3) $\mathrm{g} / \mathrm{d}$; NS).

At birth, male offspring of $\mathrm{L}$ mothers exhibited lower body weights compared with male offspring of $\mathrm{C}$ mothers $(\mathrm{C}, 7.7$ (SEM 0.1); L, 6.8 (SEM 0.2) g; $P<0.01$ ). However, there were no significant differences in body weight among the various dietary groups at $60 \mathrm{~d}$ (Cc, 406 (SEM 9); Lc, 386 (SEM 10); Ll, 384 (SEM 11) g, NS). Further, because daily food intake in all groups was similar (Cc, 22.6 (SEM 0.8); Lc, 22.4 (SEM 0.6); Ll, $22.0($ SEM 0.8$) \mathrm{g} / \mathrm{d}$; NS), it was not necessary to pair feed control rats.

No differences were observed in kidney, plasma and faeces $\mathrm{Zn}$ content between $\mathrm{Cc}$ and $\mathrm{Lc}$ groups. Ll animals showed lower $\mathrm{Zn}$ concentrations in kidneys (16.2 (SEM 1.0) $\mu \mathrm{g} / \mathrm{g}$ tissue) than both the Cc animals (26.6 (SEM 0.9) $\mu \mathrm{g} / \mathrm{g}$ tissue; $P<0.05$ ) and the Lc animals (24.9 (SEM 0.8) $\mu \mathrm{g} / \mathrm{g}$ tissue; $P<0.05)$ at $60 \mathrm{~d}$ after weaning. They also had lower plasma Zn concentrations $(0.95$ (SEM 0.09) $\mathrm{mg} / \mathrm{l})$ than the Cc animals (1.62 (SEM 0.10) $\mathrm{mg} / \mathrm{l} ; P<0.05$ ) and the Lc animals $(1.45$ (SEM $0 \cdot 10) \mathrm{mg} / \mathrm{l} ; P<0 \cdot 05)$. Similarly, faeces concentrations of $\mathrm{Zn}$ were lower in the $\mathrm{Ll}$ group (139 (SEM 16) $\mu \mathrm{g} / \mathrm{d})$ than in the Cc group (656 (SEM 105) $\mu \mathrm{g} / \mathrm{d} ; P<0.05)$ and the Lc group (586 (SEM 98) $\mu \mathrm{g} / \mathrm{d} ; P<0.05$ ).

Animals exposed to a moderately Zn-deficient diet during pre-weaning and/or post-weaning growth (L1, Lc groups) exhibited higher values $(P<0 \cdot 01)$ of SBP at the end of the dietary treatment compared with the $\mathrm{Cc}$ group (Cc, 129 (SEM 2); Lc, 148 (SEM 5); Ll, 149 (SEM 6) mmHg). However, there were no significant differences in SBP levels among the $\mathrm{Ll}$ and Lc dietary groups. Moreover, an inverse correlation was observed between birth weight and SBP ( $r$ 0.8305; $P<0.001)$ at $60 \mathrm{~d}$.

Lipid profile is shown in Table 2. Serum TAG concentration was higher in $\mathrm{Ll}$ animals compared with the $\mathrm{Cc}$ and Lc groups. However, total cholesterol, HDL-cholesterol, non-HDLcholesterol and VLDL-cholesterol concentrations were not altered by the dietary treatments.

Animals exposed to $\mathrm{Zn}$ deficiency showed lower levels of urinary NOx than $\mathrm{Cc}$ rats at $60 \mathrm{~d}$. However, Lc rats showed higher urinary NOx compared with the Ll group. Ll rats had lower urinary NOx (0.69 (SEM 0.11) nmol/ml $\times \min \times 100 \mathrm{~g})$ than both the Cc $(1.45(\mathrm{SEM} 0 \cdot 14) \mathrm{nmol} / \mathrm{ml}$ $\times \min \times 100 \mathrm{~g} ; \quad P<0.01)$ and the Lc $(1.14 \quad($ SEM 0.06$)$ 
Table 2. Effects of low-zinc diet during fetal life, lactation and post-weaning growth on lipid profile at $56 \mathrm{~d} \neq$ (Mean values with their standard errors)

\begin{tabular}{|c|c|c|c|c|c|c|}
\hline \multirow[t]{2}{*}{ Diet group... } & \multicolumn{2}{|c|}{ Cc $(n 10)$} & \multicolumn{2}{|c|}{ Lc $(n 10)$} & \multicolumn{2}{|c|}{ LI $(n 10)$} \\
\hline & Mean & SEM & Mean & SEM & Mean & SEM \\
\hline TAG (mg/l) & 1210 & 70 & 1120 & 90 & $1690^{*} \dagger$ & 90 \\
\hline Total cholesterol (mg/l) & 620 & 30 & 640 & 30 & 600 & 20 \\
\hline HDL-cholesterol (mg/l) & 490 & 40 & 560 & 60 & 460 & 30 \\
\hline Non-HDL-cholesterol (mg/l) & 160 & 30 & 190 & 20 & 180 & 10 \\
\hline VLDL-cholesterol (mg/l) & 180 & 20 & 200 & 10 & 200 & 10 \\
\hline
\end{tabular}

Cc, control Zn during pregnancy, lactation and post-weaning; Lc, low Zn during pregnancy and lactation, control Zn post-weaning; LI, low Zn during pregnancy, lactation and post-weaning.

* Mean value was significantly different from that of the Cc group $(P<0.05)$.

† Mean value was significantly different from that of the Lc group $(P<0.05)$

†TAG were analysed by the non-parametric Dunn test for multiple comparisons and the other parameters were analysed using one-way ANOVA followed by a Bonferroni post hoc test.

$\mathrm{nmol} / \mathrm{ml} \times \min \times 100 \mathrm{~g} ; P<0 \cdot 01)$ rats. Lc animals exhibited lower levels of urinary NOx than Cc animals $(P<0 \cdot 01)$.

Fig. 1 shows NOS activity, determined with the $\mathrm{L}-\left[\mathrm{U}-{ }^{14} \mathrm{C}\right]$ arginine in vitro method, in the thoracic aorta and the renal cortex. Renal and vascular tissues from the $\mathrm{Lc}$ and $\mathrm{Ll}$ groups showed decreased NOS activity compared with Cc rats. The $\mathrm{Ll}$ and $\mathrm{Lc}$ groups also exhibited lower levels $(P<0.01)$ of GFR at the end of the dietary treatment compared with the Cc group (Cc, 0.72 (SEM 0.09); Lc, 0.39 (SEM 0.01); $\mathrm{Ll}, \quad 0 \cdot 28(\mathrm{SEM} 0.03) \mathrm{ml} / \mathrm{min} \times 100 \mathrm{~g})$. Moreover, a positive correlation was observed between NOS activity in the renal cortex and GFR $(r 0.6131 ; P<0.001)$.

Table 3 and Fig. 2 show NOS activity in the thoracic aorta, intestinal arterioles and renal cortex, evaluated by the NADPH-d activity technique. NADPH-d staining in the endothelium and smooth muscle sections of the aorta and arterioles was less intense in the $\mathrm{Lc}$ and $\mathrm{Ll}$ rats than in the Cc group. Animals exposed to $\mathrm{Zn}$ deficiency also showed lower NADPH-d activity in glomeruli, proximal tubules, distal tubules and collecting ducts compared with the $\mathrm{Cc}$ group. The data are in accordance with the results of NOS activity obtained with the $\mathrm{L}-\left[\mathrm{U}_{-}{ }^{14} \mathrm{C}\right]$ arginine in vitro method.

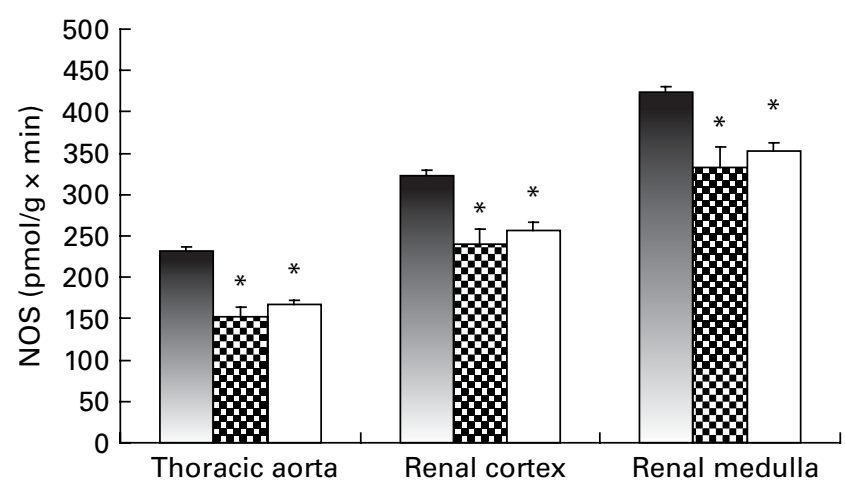

Fig. 1. NO synthase (NOS) activity measured, using L-[U- $\left.{ }^{14} \mathrm{C}\right]$ arginine as the substrate, in thoracic aorta and renal cortex of Control control (Cc, $\mathbf{\square})$, Low control (Lc, $\mathbf{B}$ ) and Low low (LI, $\square$ ) Zn diet groups. Values are means ( $n 10$ per group), with their standard errors represented by vertical bars. Data were analysed by one-way ANOVA followed by a Bonferroni post hoc test. * Mean value was significantly different from that of the Cc group $(P<0.001)$.
NOS expression was measured by Western blot analysis in the thoracic aorta and renal cortex. There was no difference in aortic nNOS protein abundance among the groups (Fig. 3(a)), and the aortic iNOS isoform was undetectable. Fig. 3(b) shows that eNOS expression was decreased in the thoracic aorta of $\mathrm{Lc}$ and $\mathrm{Ll}$ adult rats compared with the $\mathrm{Cc}$ group. All NOS isoforms were expressed in the renal cortex of Cc, Lc and L1 adult rats. Nevertheless, Zn dietary restriction during fetal life, lactation and post-weaning growth did not modify abundance of any of the NOS isoforms (Fig. 4(a)-(c)).

\section{Discussion}

In the present study we offer evidence that moderate $\mathrm{Zn}$ restriction during fetal life and postnatal growth induces alterations in lipid metabolism and in the vascular and renal NO system which could contribute to cardiovascular and renal alterations in adult life.

Animals exposed to moderate $\mathrm{Zn}$ deficiency during fetal life showed lower body weight at birth and higher BP levels in adult life. These results are in agreement with our previous study that used the same experimental design and showed that rats exposed to moderate $\mathrm{Zn}$ deficiency during fetal life and postnatal growth exhibited an increase in SBP and lower body weight at the time of weaning ${ }^{(9)}$. Therefore, the present and our previous results demonstrate the reproducibility of our model and support the hypothesis that $\mathrm{Zn}$ deficiency could be a risk factor for the development of CVD in adult life. Moreover, the close negative correlation between birth weight and SBP at $60 \mathrm{~d}$, observed in the present study, is in accordance with many epidemiological studies proposing that factors present in the prenatal environment are responsible for in utero programming of $\mathrm{CVD}^{(28-30)}$.

The 'fetal origins hypothesis' suggests that intra-uterine growth restriction results in low birth weight, programs the development of organs involved in BP regulation, such as the kidney and vessels, and may predispose to long-term health problems ${ }^{(31)}$. In this regard, we demonstrated in the present study that $\mathrm{Zn}$ restriction during fetal and early postnatal life induced vascular and renal NO system impairment in adult life. Lower NOS activity was observed in different nephron segments and in the endothelium and smooth muscle of the aorta artery and resistance arterioles, even when renal 
Table 3. NADPH diaphorase activity (measured as optical density) in thoracic aorta, intestinal arterioles and nephron segments at $60 \mathrm{~d}$ after weaning $\dagger$

(Mean values with their standard errors)

\begin{tabular}{|c|c|c|c|c|c|c|}
\hline \multirow[t]{2}{*}{ Diet group... } & \multicolumn{2}{|c|}{ Cc $(n 10)$} & \multicolumn{2}{|c|}{$\operatorname{Lc}(n 10)$} & \multicolumn{2}{|c|}{$\mathrm{LI}(n$ 10) } \\
\hline & Mean & SEM & Mean & SEM & Mean & SEM \\
\hline \multicolumn{7}{|l|}{ Thoracic aorta } \\
\hline Endothelium & 0.142 & 0.011 & $0.099^{*}$ & 0.006 & $0.089^{*}$ & 0.008 \\
\hline Smooth muscle & 0.306 & 0.011 & $0.237^{*}$ & 0.010 & $0 \cdot 215^{\star}$ & 0.005 \\
\hline \multicolumn{7}{|l|}{ Intestinal arterioles } \\
\hline Endothelium & $0 \cdot 240$ & 0.006 & $0 \cdot 104^{*}$ & 0.007 & $0 \cdot 115^{\star}$ & 0.009 \\
\hline Smooth muscle & 0.284 & 0.007 & $0.216^{*}$ & 0.006 & $0.205^{*}$ & 0.005 \\
\hline \multicolumn{7}{|l|}{ Nephron segments } \\
\hline Glomeruli & 0.162 & 0.006 & $0 \cdot 126^{*}$ & 0.009 & $0 \cdot 105^{\star}$ & 0.009 \\
\hline Proximal tubule & 0.250 & 0.008 & $0.201^{*}$ & 0.006 & $0.194^{\star}$ & 0.005 \\
\hline Distal tubule & 0.246 & 0.007 & $0 \cdot 140^{\star}$ & 0.009 & $0 \cdot 134^{\star}$ & 0.008 \\
\hline Collecting duct & 0.208 & 0.006 & $0.156^{*}$ & 0.007 & $0 \cdot 158^{\star}$ & 0.007 \\
\hline
\end{tabular}

Cc, control Zn during pregnancy, lactation and post-weaning; Lc, low Zn during pregnancy and lactation, control Zn post-weaning; LI, low Zn during pregnancy, lactation and post-weaning.

${ }^{*}$ Mean value was significantly different from that of the Cc group $(P<0.001)$

† Data were analysed using one-way ANOVA followed by a Bonferroni post hoc test.

and plasma $\mathrm{Zn}$ content was restored through an adequate-Zn diet during post-weaning growth. Additionally, animals exposed to $\mathrm{Zn}$ restriction showed lower urinary excretion of NO endproducts, whose concentration is an indicator of systemic NO production and/or bioavailability. Therefore, the above findings suggest that $\mathrm{Zn}$ deficiency in different periods of growth can impair NO production.
The reduction in vascular NOS activity, regardless of the period of growth, is associated with a lower protein expression of the eNOS isoform. Therefore, the lower eNOS-derived NO production in conduct arteries would impair vascular smooth muscle relaxation and would not allow the response to increases in flow and in shear stress, inducing a decrease in arterial compliance ${ }^{(32-34)}$ (a)

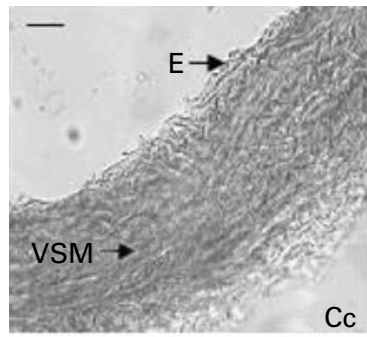

(b)

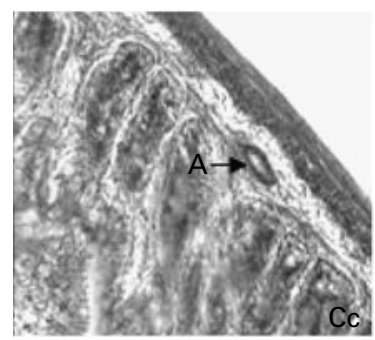

(c)

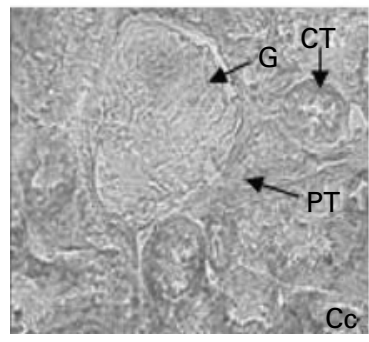

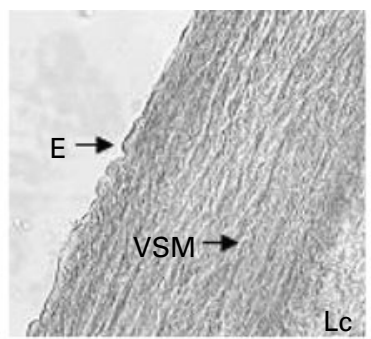
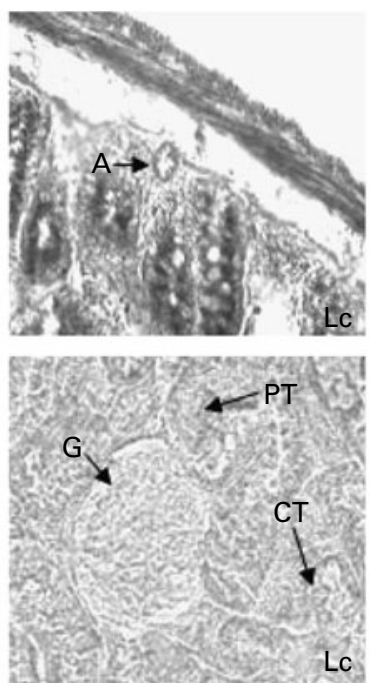
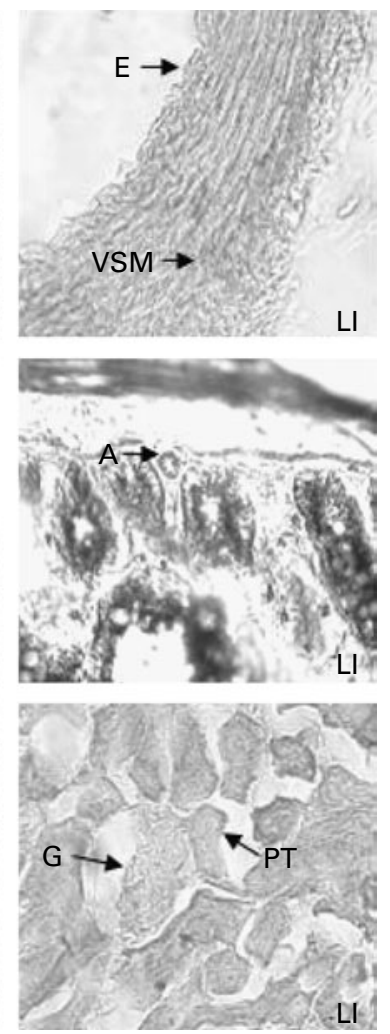

Fig. 2. NADPH-diaphorase staining in (a) thoracic aorta, (b) intestinal arterioles and (c) renal cortex of Control control (Cc), Low control (LC) and Low low (LI) $\mathrm{Zn}$ diet groups at the end of the experimental protocol ( $n 10$ for each group). Arrows indicate staining in endothelium (E), vascular smooth muscle (VSM), intestinal arterioles (A), glomeruli (G), proximal tubule (PT) and collecting tubule (CT). All images are at the same magnification of $\times 400$. Scale bar $=30 \mu \mathrm{m}$. 
(a)

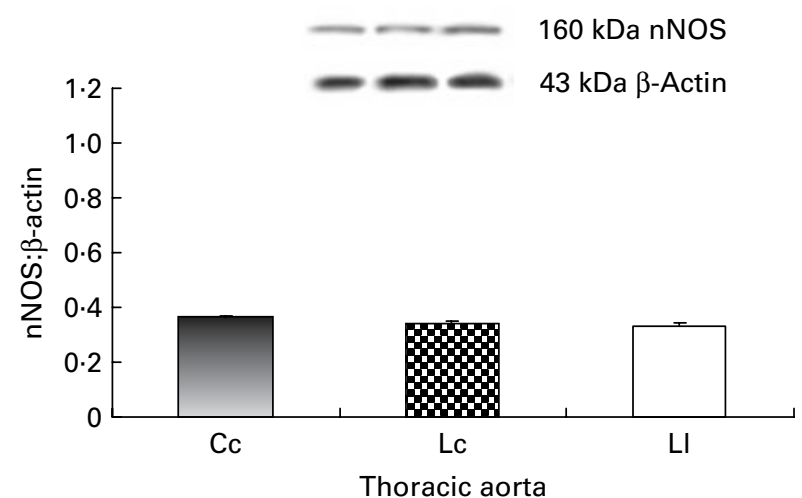

(b)

Meanwhile, in the kidney, reduced NOS activity in the renal cortex could be one of the possible mechanisms involved in the decrease in GFR that we observed in Zn-deficient animals. This hypothesis is supported by the correlation observed between NOS activity in the renal cortex and GFR, as well as by evidence showing that NO plays a major role in the maintenance of renal perfusion, GFR and low renal vascular resistance in the kidney ${ }^{(14,15)}$. Moreover, reduced renal NO production could also be associated with morphological renal alterations, proteinuria, renal fibrosis and apoptosis, leading to progressive hypertension and severe renal injury in adult $\mathrm{Zn}$-deficient rats $^{(9,12)}$.

Restitution of renal $\mathrm{Zn}$ content by an adequate- $\mathrm{Zn}$ diet during post-weaning growth was not enough to normalise BP and GFR, probably due to irreversible alterations induced in utero, such as renal and vascular NO system impairment as well as the morphological renal alterations previously reported $^{(9)}$.

Contrasting with the aorta artery, the decrease in renal NOS activity was not associated with lower expression of NOS isoform proteins. Contrasting information about NOS

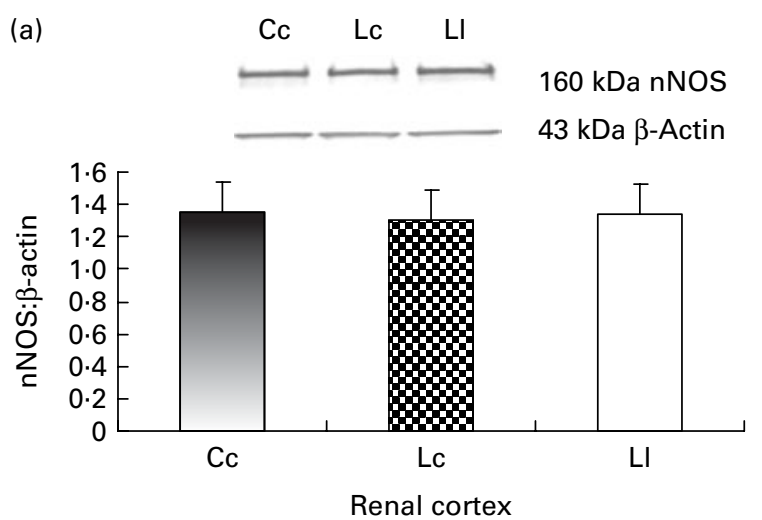

(b)
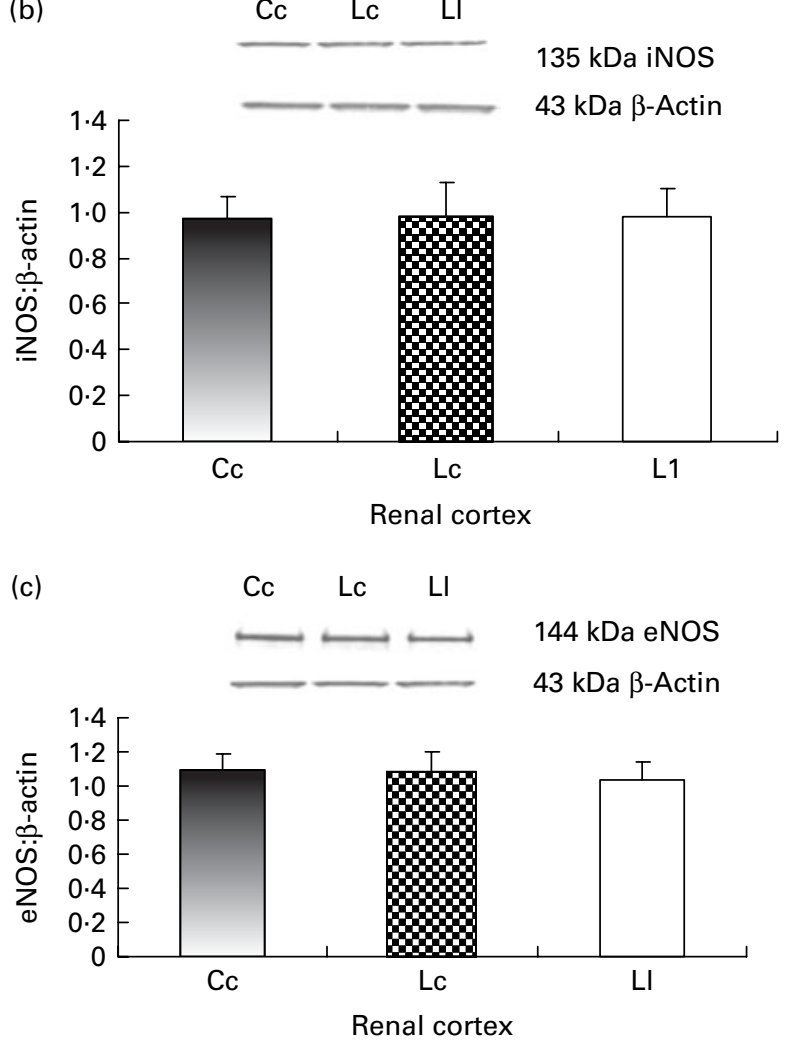

Fig. 4. Western immunoblots showing the protein expression of (a) neuronal NO synthase (nNOS), (b) inducible NOS(iNOS) and (c) endothelial NOS (eNOS) in the renal cortex of Control control (Cc, $\mathbf{\square})$, Low control (Lc, B) and Low low (LI, $\square)$ Zn diet groups. All experiments were performed in triplicate. Each blot was normalised to expression of the $\beta$-actin marker from the same gel. Values are means ( $n 10$ per group), with their standard errors represented by vertical bars. Data were analysed by one-way ANOVA followed by a Bonferroni post hoc test.

isoform expression in different tissues of animals exposed to $\mathrm{Zn}$ deficiency is found in the literature. In weanling rats exposed to severe $\mathrm{Zn}$ deficiency, iNOS expression has been induced in the lung, skin and intestine ${ }^{(35-37)}$. On the other hand, Sato et al. found that severe $\mathrm{Zn}$ deficiency during adult life does not change NOS activity and aortic eNOS expression in normotensive rats ${ }^{(38)}$ and enhances expression of eNOS mRNA and protein in the thoracic aorta of spontaneously hypertensive rats ${ }^{(39)}$.

Taking into account our evidence and that from other reports, we suggest that the enhanced oxidative stress 
condition would probably contribute to decreased NOS activity, since oxygen free radicals could trigger the uncoupling of the enzyme ${ }^{(9,16)}$. In addition, we postulate that disturbances in $\mathrm{Zn}$ homeostasis during critical periods of growth would affect other regulation enzymic mechanisms, including alterations in the dimeric enzyme structure, in substrate and cofactor synthesis and transport, and/or in the activity of humoral and nervous factors ${ }^{(40,41)}$.

On the other hand, animals exposed to $\mathrm{Zn}$ restriction since fetal life and until adulthood showed augmented plasma TAG level, another cardiovascular risk factor. The increase in TAG level seems to be induced mainly by $\mathrm{Zn}$ dietary restriction during post-weaning growth. The present results are in agreement with previous work on severe $\mathrm{Zn}$ deficiency in adult rats ${ }^{(17,18)}$. However, we did not observe changes in total cholesterol and in the other major serum lipoprotein fractions as observed in experimental models of severe $\mathrm{Zn}$ deficiency ${ }^{(19-22)}$. These contrasting observations may indicate that the effects of $\mathrm{Zn}$ deficiency on lipid profile may be related to the duration of the low $\mathrm{Zn}$ dietary treatment, the degree of $\mathrm{Zn}$ restriction, the period of life involved and the environment.

In conclusion, the results of the present study give strong evidences that $\mathrm{Zn}$ deficiency during intra-uterine and postnatal growth can result in low birth weight and in the programming of renal and vascular NO system dysfunction. These alterations could be linked to the elevated BP observed in adult life.

\section{Acknowledgements}

The present study was supported by grants PIP11220080101121/09 CONICET and PICT 12126 FONCYT, Secretary of Science and Technology, Argentina and by grants B026 and B008 from the University of Buenos Aires and IQUIMEFA-CONICET, Argentina.

The authors thank Sebastián Finella (Fisiología, Facultad de Farmacia y Bioquímica, Universidad de Buenos Aires) and Adriana Ruth Weisstaub (Nutrición, Facultad de Farmacia y Bioquímica, Universidad de Buenos Aires) for their technical assistance and Ana Borthwick for English language proofreading.

A. T. performed the experiments and participated in experimental design, data analysis and in writing the manuscript. R. E. participated in renal and vascular NOS activity determination.

V. Z. and L. S. carried out lipid analyses. A. F. carried out Western blot analysis. H. F. performed the determination of $\mathrm{Zn}$ content in plasma, faeces, kidneys and diets. A. M. B. assisted in scientific and technical supervision. M. A. C. participated in scientific and technical supervision, experimental design, data analysis and in writing the manuscript. C. A. participated in scientific and technical supervision, experimental design, data analysis and in writing the manuscript.

The authors declare that they have no conflicts of interest.

\section{References}

1. Barker DJ (1992) The fetal origins of diseases of old age. Eur J Clin Nutr 46, Suppl. 3, S3-S9.

2. McMillen IC \& Robinson JS (2005) Developmental origins of the metabolic syndrome: prediction, plasticity, and programming. Physiol Rev 85, 571-633.
3. Alexander BT (2006) Fetal programming of hypertension. Am J Physiol Regul Integr Comp Physiol 290, R1-R10.

4. Vallee BL \& Falchuk KH (1993) The biochemical basis of zinc physiology. Physiol Rev 73, 79-118.

5. Sandstead HH (1995) Is zinc deficiency a public problem? Nutrition 11, Suppl. 1, 87-92.

6. Keen CL, Hanna LA, Lanoue L, et al. (2003) Developmental consequences of trace mineral deficiencies in rodents: acute and long-term effects. J Nutr 133, Suppl. 1, 1477S-1480S.

7. Maret W \& Sandstead HH (2008) Possible roles of zinc nutriture in the fetal origins of disease. Exp Gerontol 43, $378-381$.

8. Sandström B (1997) Bioavailability of zinc. Eur J Clin Nutr 51, Suppl. 1, S17-S19.

9. Tomat AL, Inserra F, Veiras L, et al. (2008) Moderate zinc restriction during fetal and postnatal growth in rats: effects on adult arterial blood pressure and kidney. Am J Physiol Regul Integr Comp Physiol 295, R543-R549.

10. Tomat AL, Weisstaub AR, Jáuregui A, et al. (2005) Moderate zinc deficiency influences arterial blood pressure and vascular nitric oxide pathway in growing rats. Pediatr Res 58, 672-676.

11. Tomat AL, Costa MA, Girgulsky LC, et al. (2007) Zinc deficiency during growth: influence on renal function and morphology. Life Sci 80, 1292-1302.

12. Ribeiro MO, Antunes E, Nucci G, et al. (1992) Chronic inhibition of nitric synthesis, a new model of arterial hypertension. Hypertension 20, 298-303.

13. Navarro J, Sanchez A, Saiz J, et al. (1994) Hormonal, renal, and metabolic alterations during hypertension induced by chronic inhibition of NO in rats. Am J Physiol 267, R1516-R1521.

14. Kone BC (2004) Nitric oxide synthesis in the kidney: isoforms, biosynthesis, and functions in health. Semin Nephrol 24, 299-315.

15. Majid DS \& Navar LG (2001) Nitric oxide in the control of renal hemodynamics and excretory function. Am J Hypertens $14,74 S-82 S$.

16. Zou MH, Shi C \& Cohen RA (2002) Oxidation of the zincthiolate complex and uncoupling of endothelial nitric oxide synthase by peroxynitrite. J Clin Invest 109, 817-826.

17. Kettler SI, Eder K, Kettler A, et al. (2000) Zinc deficiency and the activities of lipoprotein lipase in plasma and tissues of rats force-fed diets with coconut oil or fish oil. J Nutr Biochem 11, $132-138$.

18. Daniel H \& Dieck HT (2004) Nutrient-gene interactions: a single nutrient and hundreds of target genes. Biol Chem 385, 571-583

19. Fischer PW, Giroux A, Belonje B, et al. (1980) The effect of dietary copper and zinc on cholesterol metabolism. Am J Clin Nutr 33, 1019-1025.

20. Koo SI \& Williams DA (1981) Relationship between the nutritional status of zinc and cholesterol concentration of serum lipoproteins in adult male rats. Am J Clin Nutr 34, 2376-2381.

21. Schneeman BO, Lacy D, Ney D, et al. (1986) Similar effects of zinc deficiency and restricted feeding on plasma lipids and lipoproteins in rats. $J$ Nutr 116, 1889-1895.

22. Faure P, Roussel AM, Richard MJ, et al. (1991) Effect of an acute zinc depletion on rat lipoprotein distribution and peroxidation. Biol Trace Elem Res 28, 135-146.

23. Reeves PG, Nielsen FH \& Fahey GC Jr (1993) AIN-93 purified diets for laboratory rodents: final report of the American Institute of Nutrition ad hoc Writing Committee on the reformulation of the AIN-76A rodent diet. J Nutr 123, 1939-1951.

24. Assman G, Jabs H, Kohnert U, et al. (1984) LDL-cholesterol determination in blood serum following precipitation of LDL with polyvinylsulfate. Clin Chim Acta 140, 77-83. 
25. Verdon CP, Burton BA \& Prior RL (1995) Sample pretreatment with nitrate reductase and glucose-6-phosphate dehydrogenase quantitatively reduces nitrate while avoiding interference by NADP + when the Griess reaction is used to assay for nitrite. Anal Biochem 224, 502-508.

26. Costa MA, Loria A, Elesgaray R, et al. (2004) Role of nitric oxide pathway in hypotensive and renal effects of furosemide during extracellular volume expansion. J Hypertens 22, 1561-1569.

27. Rothe F, Canzler U \& Wolf G (1998) Subcellular localization of the neuronal isoform of nitric oxide synthase in the rat brain: a critical evaluation. Neuroscience 83, 259-269.

28. Eriksson J, Forsén T, Tuomilehto J, et al. (2000) Fetal and childhood growth and hypertension in adult life. Hypertension 36, 790-794.

29. Godfrey KM \& Barker DJ (2000) Fetal nutrition and adult diseases. Am J Clin Nutr 71, Suppl. 5, 1344S-1352S.

30. Barker DJ, Forsén T, Eriksson JG, et al. (2002) Growth and living conditions in childhood and hypertension in adult life: a longitudinal study. J Hypertens 20, 1951-1956.

31. Barker DJ (1995) Fetal origins of coronary heart diseases. BMJ 311, 171-174.

32. Moncada $S$ (1997) Nitric oxide in the vasculature: physiology and pathophysiology. Ann N Y Acad Sci 15, 60-67.

33. McIntyre M, Bohr DF \& Dominiczak AF (1999) Endothelial function in hypertension: the role of superoxide anion. Hypertension 34, 539-545.
34. Vapaatalo H \& Mervaala E (2001) Clinically important factors influencing endothelial function. Med Sci Monit 7, $1075-1085$.

35. St Croix CM, Leelavaninchkul K, Watkins SC, et al. (2005) Nitric oxide and zinc homeostasis in acute lung injury. Proc Am Thorac Soc 2, 236-242.

36. Cui L, Takagi Y, Wasa M, et al. (1999) Nitric oxide synthase inhibitor attenuates intestinal damage induced by zinc deficiency in rats. $J$ Nutr 129, 792-798.

37. Cui L \& Okada A (2000) Nitric oxide and manifestations of lesions of skin and gastrointestinal tract in zinc deficiency. Curr Opin Clin Nutr Metab Care 3, 247-252.

38. Sato M, Kurihara N, Moridaira K, et al. (2003) Dietary $\mathrm{Zn}$ deficiency does not influence systemic blood pressure and vascular nitric oxide signaling in normotensive rats. Biol Trace Elem Res 91, 157-172.

39. Sato M, Yanagisawa H, Nojima Y, et al. (2002) Zn deficiency aggravates hypertension in spontaneously hypertensive rats: possible role of $\mathrm{Cu} / \mathrm{Zn}$-superoxide dismutase. Clin Exp Hypertens 24, 355-370.

40. Herrera M \& Garvin JL (2005) Recent advances in the regulation of nitric oxide in the kidney. Hypertension 45, 1062-1067.

41. Mount PF \& Power DA (2006) Nitric oxide in the kidney: functions and regulation of synthesis. Acta Physiol (Oxf) 187, $433-446$ 